\title{
FIRST DIVERTOR PHYSICS STUDIES IN WENDELSTEIN 7-X
}

T. Sunn Pedersen ${ }^{1,2}$, R. König ${ }^{1}$, M. Jakubowski ${ }^{1,17}$, M. Krychowiak ${ }^{1}$, D. Gradic ${ }^{1}$, C. Killer ${ }^{1}$, H. Niemann ${ }^{1}$, T. Szepesi ${ }^{6}$, U. Wenzel ${ }^{1}$, A. Ali ${ }^{1}$, G. Anda ${ }^{6}$, J. Baldzuhn ${ }^{1}$, T. Barbui ${ }^{5}$, C. Biedermann $^{1}$, B. D. Blackwell ${ }^{3}$, H.-S. Bosch ${ }^{1}$, S. Bozhenkov ${ }^{1}$, R. Brakel ${ }^{1}$, S. Brezinsek ${ }^{4}$, J. $^{2}$ $\mathrm{Cai}^{4}$, B. Cannas ${ }^{18}$, J.W. Coenen ${ }^{4}$, J. Cosfeld ${ }^{4}$, A. Dinklage ${ }^{1}$, T. Dittmar ${ }^{4}$, P. Drewelow ${ }^{1}$, P. Drews $^{4}$, D. Dunai ${ }^{6}$, F. Effenberg ${ }^{5}$, M. Endler ${ }^{1}$, Y. Feng ${ }^{1}$, J. Fellinger ${ }^{1}$, O. Ford ${ }^{1}$, H. Frerichs ${ }^{5}$, G. Fuchert ${ }^{1}$, Y. Gao ${ }^{4}$, J. Geiger ${ }^{1}$, A. Goriaev ${ }^{13,14}$, K. Hammond ${ }^{1}$, J. Harris ${ }^{9}$, D. Hathiramani ${ }^{1}$, M. Henkel ${ }^{4}$, Ye. O. Kazakov ${ }^{13}$, A. Kirschner ${ }^{4}$, A. Knieps ${ }^{4}$, M. Kobayashi ${ }^{11}$; G. Kocsis ${ }^{6}$, P.

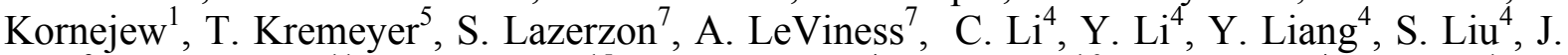
Lore $^{9}$, S. Masuzaki ${ }^{11}$, V. Moncada ${ }^{15}$, O. Neubauer ${ }^{4}$, T. T. Ngo ${ }^{16}$, J. Oelmann ${ }^{4}$, M. Otte ${ }^{1}$, V. Perseo $^{1}$, F. Pisano ${ }^{8}$, A. Puig Sitjes ${ }^{1}$, M. Rack ${ }^{4}$, M. Rasinski ${ }^{4}$, J. Romazanov ${ }^{4}$, L. Rudischhauser $^{1}$, G. Schlisio ${ }^{1}$, J.C. Schmitt ${ }^{7}$, O. Schmitz ${ }^{5}$, B. Schweer ${ }^{4}$, S. Sereda ${ }^{4}$, M.

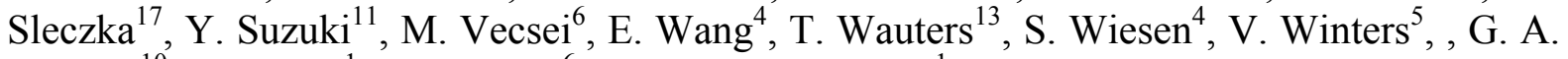
Wurden $^{10}$, D. Zhang ${ }^{1}$, S. Zoletnik ${ }^{6}$, and the W7-X Team ${ }^{1}$

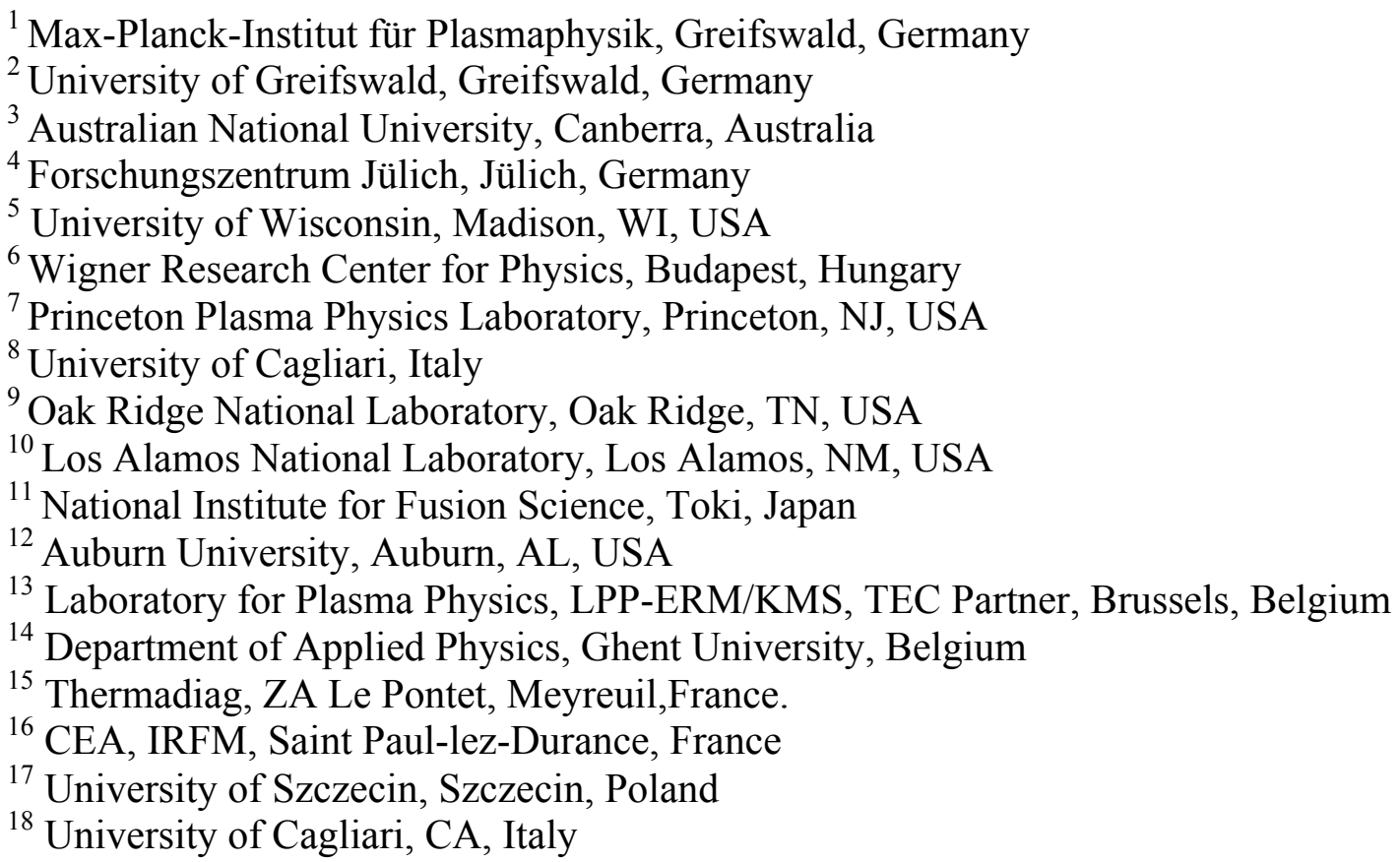

\section{Abstract}

The Wendelstein 7-X (W7-X) optimized stellarator fusion experiment, which went into operation in 2015, has been operating since 2017 with an un-cooled modular graphite divertor. This allowed first divertor physics studies to be performed at pulse energies up to $80 \mathrm{MJ}$, as opposed to $4 \mathrm{MJ}$ in the first operation phase, where five inboard limiters were installed instead of a divertor. This, and a number of other upgrades to the device capabilities, allowed extension into regimes of higher plasma density, heating power, and performance overall, e.g. setting a new stellarator world record triple product. The paper focuses on the first physics studies of how the island divertor works. The plasma heat loads arrive to a very high degree on the divertor plates, with only minor heat loads seen on other components, in particular baffle structures built in to aid neutral compression. The strike line shapes and locations change significantly from one magnetic configuration to another, in very much the same way that codes had predicted they would. Strike-line widths are as large as $10 \mathrm{~cm}$, and the wetted areas also large, up to about $1.5 \mathrm{~m}^{2}$, which bodes well for future operation phases. Peak local heat loads onto the divertor were in general benign and project below the $10 \mathrm{MW} / \mathrm{m}^{2}$ limit of the future water-cooled divertor when operated with $10 \mathrm{MW}$ of heating power, with the exception of low-density attached operation in the high-iota 
configuration. The most notable result was the complete (in all 10 divertor units) heat-flux detachment obtained at highdensity operation in hydrogen.

\section{INTRODUCTION}

Wendelstein 7-X (W7-X) went successfully into operation in 2015 [1-4]. With a 30 cubic meter volume, a superconducting coil system operating at $2.5 \mathrm{~T}$, and steady-state heating capability of up to $10 \mathrm{MW}$, it was built to demonstrate the benefits of optimized stellarators at parameters approaching those of a fusion power plant. Operation phase 1.2a (OP1.2a), which was performed in the second half of 2017, was the first operation phase with a full complement of plasma-facing components, including the full complement of 10 passively cooled fine-grain graphite divertor units, referred to collectively as the Test Divertor Unit (TDU). OP1.2a also featured an electron cyclotron resonance heating (ECRH) system with 10 gyrotrons, more than 30 diagnostic systems, and a pellet fueling system. The TDU has the same geometry as the water-cooled steady-state carbon-fiber-composite divertor that will be in operation in the early 2020's in Operation Phase 2 (OP2). The upgrades enabled significant performance extensions and a comprehensive physics program [5], specifically the start of a divertor research program, results from which are reported here. The TDU implements the so-called island divertor concept, where large intrinsic island chains at the plasma edge provide multiple x-points and the possibility to intersect the outflowing plasma in locations somewhat removed from the closed flux surfaces. This concept was used successfully on the predecessor experiment W7-AS [6].

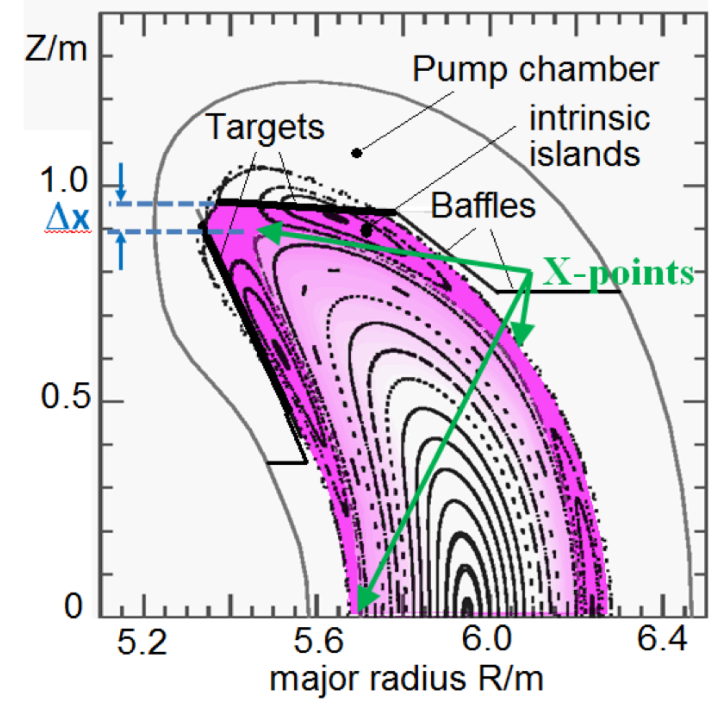

Figure 1. Poincare plot presenting the geometry of the island divertor configuration of W7-X (with two and a half islands out of five visible in the shown upper half.

The Poincaré plot for the so-called "standard" configuration is shown in Figure 1. It presents a poloidal cut through the magnetic flux surfaces of W7-X with 2 (and a half) out of 5 islands visible. These two islands intersect divertor target plates for a certain range of toroidal angles, thus forming heat and particle exhaust channels. Because the stellarator, and in particular the edge island boundary, is inherently three-dimensional, sophisticated diagnostics and codes are required to understand the plasma boundary. One advantage is the long connection lengths, which arises due to the low magnetic shear in the "Wendelstein 7"line of stellarators. The pitch of field lines in the divertor area is much smaller in the case of W7-X than in conventional diverted tokamaks. As will be shown below, this allows for very efficient heat flux spreading on the divertor over a wide range of plasma parameters.

\section{ATTACHED DIVERTOR OPERATION AT LOWER DENSITIES}




\subsection{Divertor heat load patterns}

At low to medium densities ( $\mathrm{n}_{\mathrm{e}}$ up to about $4 * 10^{19} \mathrm{~m}^{-3}$ ), the plasmas were attached. More than $95 \%$ of the heat loads exiting the scrape-off layer (SOL) landed on the 10 divertor plates. The power load distribution on the divertor surface is determined by the intersection of the island chain forming the island divertor with the divertor target plates, and therefore the 3D strike-line geometry strongly depends on the chosen magnetic configuration. Application of error field correction brings the measured strike line geometry into good agreement with numerical predictions $[7,8]$. An example for the standard configuration is shown in Fig. 2. It shows the heat flux distribution measured on the surface of the lower divertor in module 2. On W7-X, the divertor surface temperatures are measured by $10 \mathrm{IR}$ cameras (one for each divertor unit) detecting in the IR wavelength ranges of either $8-10 \mu \mathrm{m}$ or 3-5 $\mu \mathrm{m}$. The heat flux is then determined with the THEODOR code [9] by solving a twodimensional heat diffusion equation for the bulk of the tile with the surface temperature time evolution as the input.
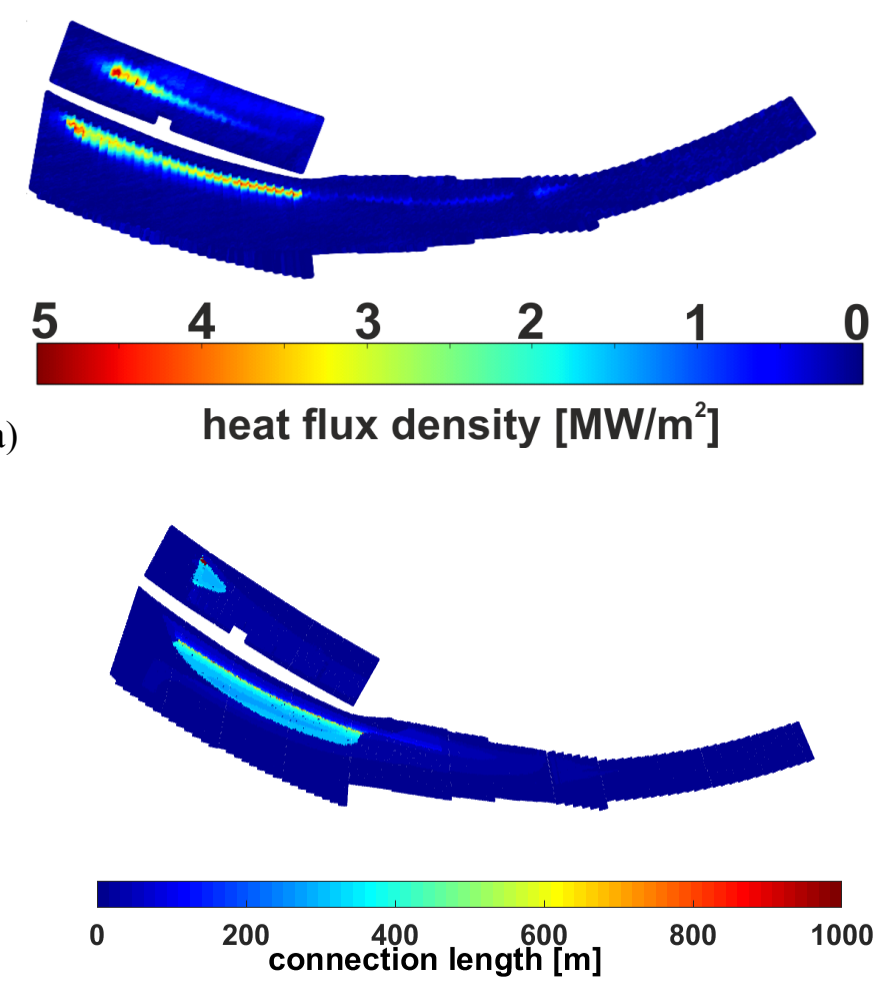

(b)

Figure 2. (a) Heat flux density measured in one out of ten divertors of W7-X in so-called standard configuration. Two strike lines are visible on the horizontal (lower) and vertical (upper) target modules.(b)Calculated magnetic footprint plot for standard configuration showing structure of magnetic field lines intersecting divertor target plates. Two strike lines are formed by field lines with connection length of a few hundred meters.

The position of a strike line is primarily defined by the intersection of target plates with large edge magnetic islands. The distribution of the heat flux within the strike line and its shape depends on several factors, e.g. field line connection length, plasma density and power entering the scrape-off layer. An example of the effects of plasma density on the strike line shape is shown in Figure 3, where the peaking factor of the strike line is plotted against the plasma line integrated density measured by interferometry. The peaking factor is defined as follows: A local peaking factor is determined by going along a line perpendicular to the strike line itself, taking the ratio of the largest measured heat flux along the line, to the average heat flux in the wetted region. The wetted region is defined as that with heat loads above approximately $0.15-0.2 \mathrm{MW} / \mathrm{m}^{2}$, roughly the detection limit of the infrared observation 
system. These local peaking factors are then averaged over the full strike line to yield the overall peaking factor shown in the Figure. Additionally, the input power for each discharge is shown in the plot as the color of each dot. Increasing the density leads to smaller peaking factor, indicating that the power reaching the divertor is spread more uniformly on its surface. As W7-X aims to operate at very high densities $\left(\mathrm{n}_{\mathrm{e}}>10^{20} \mathrm{~m}^{-3}\right)$, this is a beneficial trend in terms of the safety of the plasma facing components. Interestingly, increasing the input power (and by that increasing the power entering into the SOL, $\mathrm{P}_{\mathrm{SOL}}$ ) also appears to result in a lower peaking factor. This suggests that increasing $P_{\mathrm{SOL}}$ leads to more efficient spreading of the power loads. More information about the upstream SOL parameters and their dependence on heating power and density can be found in [10].

An important parameter in connection with power load spreading is the exponential power fall-off length in the radial direction at the outboard midplane, $\lambda_{\mathrm{q}}$. As reported elsewhere [10] $\lambda_{\mathrm{q}}$ values have been measured at the outboard midplane with reciprocating Langmuir probes, and are typically $9-14 \mathrm{~mm}$ for attached divertor operation. This means that $\lambda_{\mathrm{q}}$ in the island divertor does not directly fit the Eich-type scaling of tokamaks, which is usually based on divertor heat load measurements mapped back along the magnetic field to the outboard midplane [11]:

$\lambda_{\mathrm{q}, \text { Eich }}[\mathrm{mm}]=0.63 * \mathrm{~B}_{\mathrm{pa}}{ }^{-1.19} \quad$ Eq. 1

Here $\mathrm{B}_{\mathrm{pa}}$ is the poloidal magnetic field in Tesla at the outboard midplane. In W7-X, $\mathrm{B}_{\mathrm{pa}}$ is about $0.22 \mathrm{~T}$, yielding a predicted $\lambda_{\mathrm{q}}$, Eich $=3.8 \mathrm{~mm}$, about three times smaller than the actual measured values. The much wider SOL is an encouraging observation but should not be taken as the final result on this issue. In particular, Eq. 1 is for tokamak H-modes, and a clear tokamak-like H-mode has not yet been identified in W7-X.

It is also not clear yet to what degree our results are consistent with the fundamental assumptions of the heuristic model of Goldston [12] which imply that $\lambda_{\mathrm{q}}$ is proportional to the scrape-off layer connection length $\mathrm{L}_{\mathrm{c}}$. In the stellarator island divertor the poloidal Bfield, $B_{p}$, is not inversely proportional to the $S O L$ connection length, $\mathrm{L}_{c}$. $\mathrm{L}_{\mathrm{c}}$ depends primarily on the magnetic shear, and not on the magnitude of $\mathrm{B}_{\mathrm{pa}}$, and $\mathrm{L}_{\mathrm{c}}$ can be made very large in $\mathrm{W} 7-\mathrm{X}$, of order 100-400 $\mathrm{m}$, since it is a low-shear stellarator [13]. The experimental relationship between $\mathrm{L}_{\mathrm{c}}$ and $\lambda_{\mathrm{q}}$ are currently being analyzed, and first indications are that a linearly proportional relationship between $\lambda_{\mathrm{q}}$ and the connection length $\mathrm{L}_{\mathrm{c}}$ (which has a $1 / \mathrm{B}_{\mathrm{pa}}$ scaling in the tokamak x-point divertor but not in the stellarator island divertor) is not reproduced: $\lambda_{\mathrm{q}}$ does continue to grow with $\mathrm{L}_{\mathrm{c}}$ but slower than linearly.

A large $\lambda_{\mathrm{q}}$ is a positive result for divertor operation, and an independence of $\lambda_{\mathrm{q}}$ from $\mathrm{B}_{\mathrm{p}}$ could be of significant importance. It is known in both stellarators and tokamaks that global energy confinement scales close to linearly with $\mathrm{B}_{\mathrm{p}}$. A decoupling would mean that benign attached divertor heat loads can be combined with good core confinement in the stellarator island divertor. It is a challenge in standard x-point divertor tokamaks to combine the best core confinement performance with benign divertor heat loads, partly due to the tight coupling through $B_{p}$. More details on W7-X divertor power loads are given in [14]. 
load peaking factor (Standard configuration)

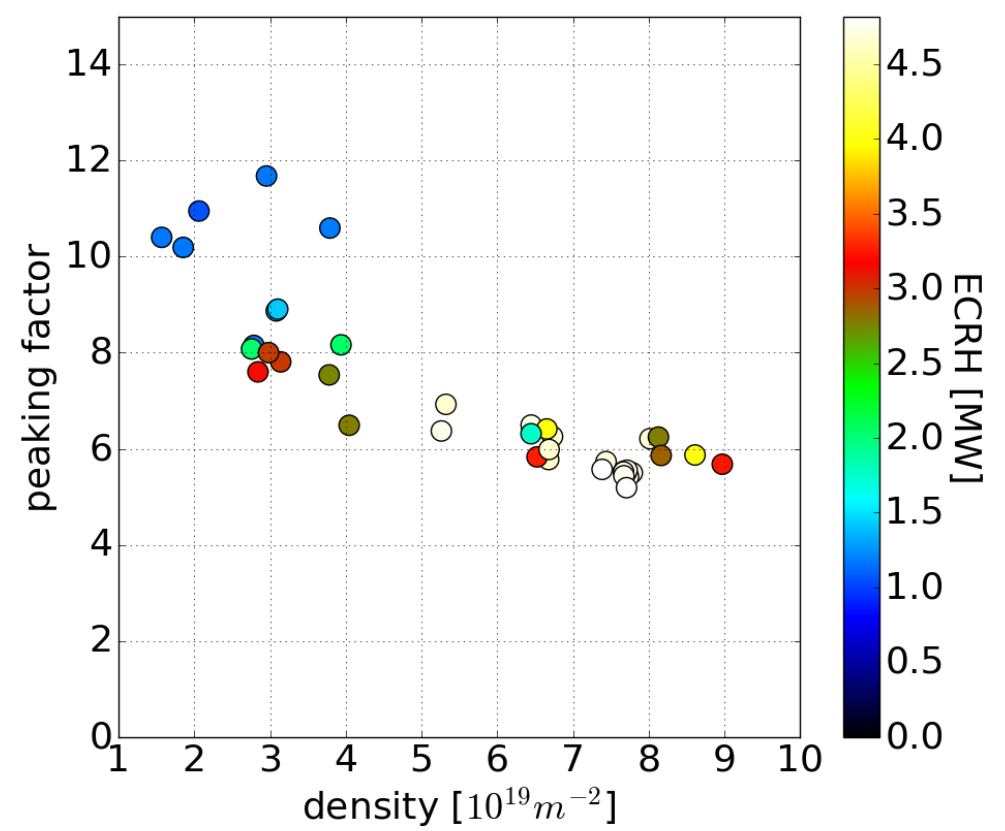

Figure 3. Change of peaking factor with plasma density for discharges with standard configuration.

\section{TESTS OF SCRAPER ELEMENTS}

The scraper elements [15] are designed to protect the edges of divertor components from overload in certain long-pulse OP2 scenarios where the magnetic topology changes due to $\sim 40 \mathrm{kA}$ of net toroidal current and $\sim 3 \%$ plasma beta [16]. As these conditions are not directly accessible in OP1.2a, a set of magnetic configurations were developed to mimic this topology change using the W7-X coil set [17]. Experiments were performed in OP1.2a, without scraper elements installed, using a series of such "mimic" configurations corresponding to five characteristic time points in the evolution of the OP2 scenario. The heat fluxes inferred from infrared camera measurements [18] show good qualitative agreement with predictions from field line diffusion calculations from the DIV3D code [19], as shown in Fig. 4. These results confirm that the scraper elements intersect field lines that connect to the divertor edges for the magnetic topology where they also carry significant plasma heat. They thus protect the divertor edges from overload. Assuming that the OP2 scenario development simulations are correct, and experiments are executed as assumed in the simulations, an overload situation would occur without scraper elements, but not with scraper elements. Alternative mitigation strategies not requiring scraper elements include electron-cyclotron current drive, using the existing set of 20 planar superconducting coils (see eg. [20]) to control the edge transform, and the development of another startup scenario that avoids the overloading but still allows reaching the desired steady-state operating point by optimizing (e.g.) the time evolution of the density and heating power. These will be reported on separately. 

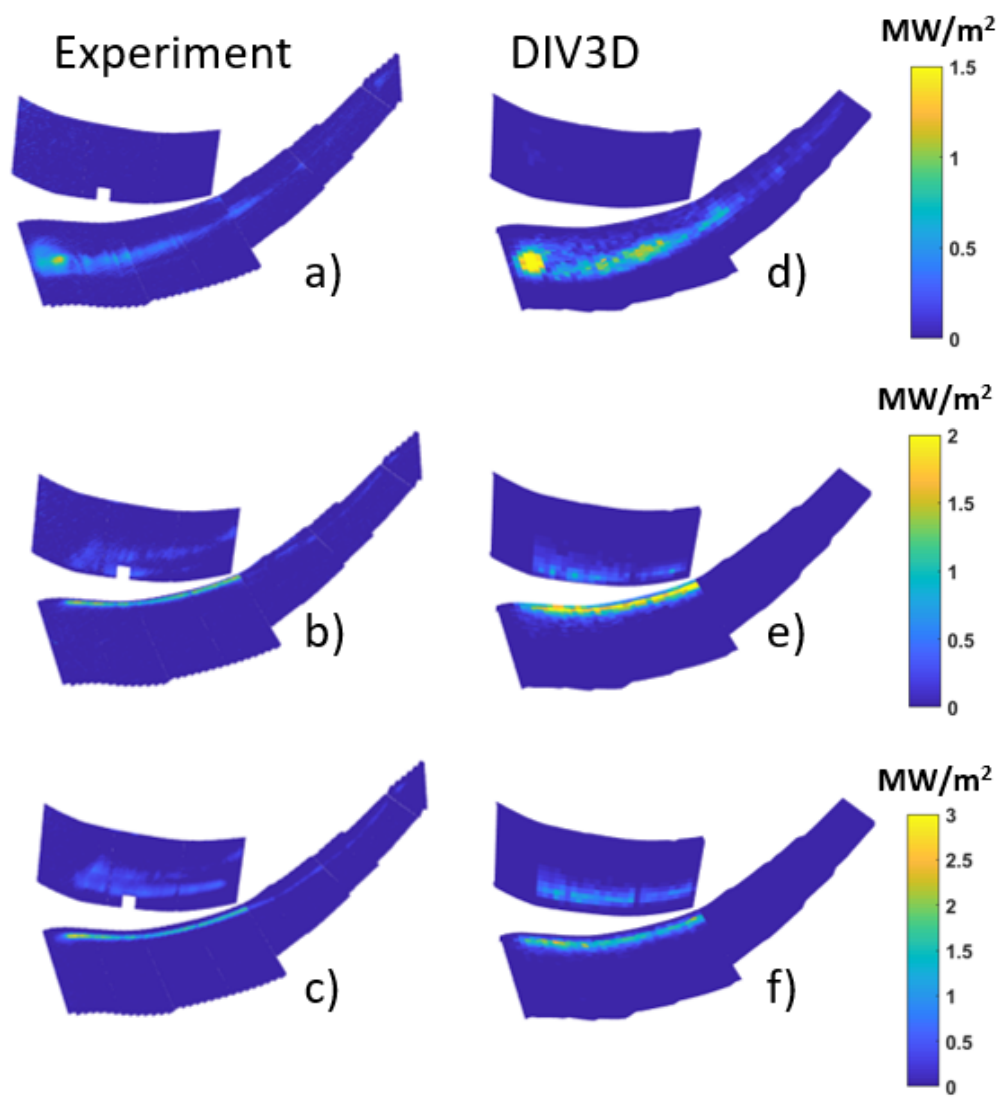

Figure 4. Heat fluxes from experiment (a-c) and DIV3D (d-e) in the 0kA (a,d), 32kA (b,e), and 43kA (c,f) mimic configurations.

\section{DETACHMENT}

\subsection{General observations}

A power detachment was observed in medium-density hydrogen discharges. An example is shown in Fig. 5. 


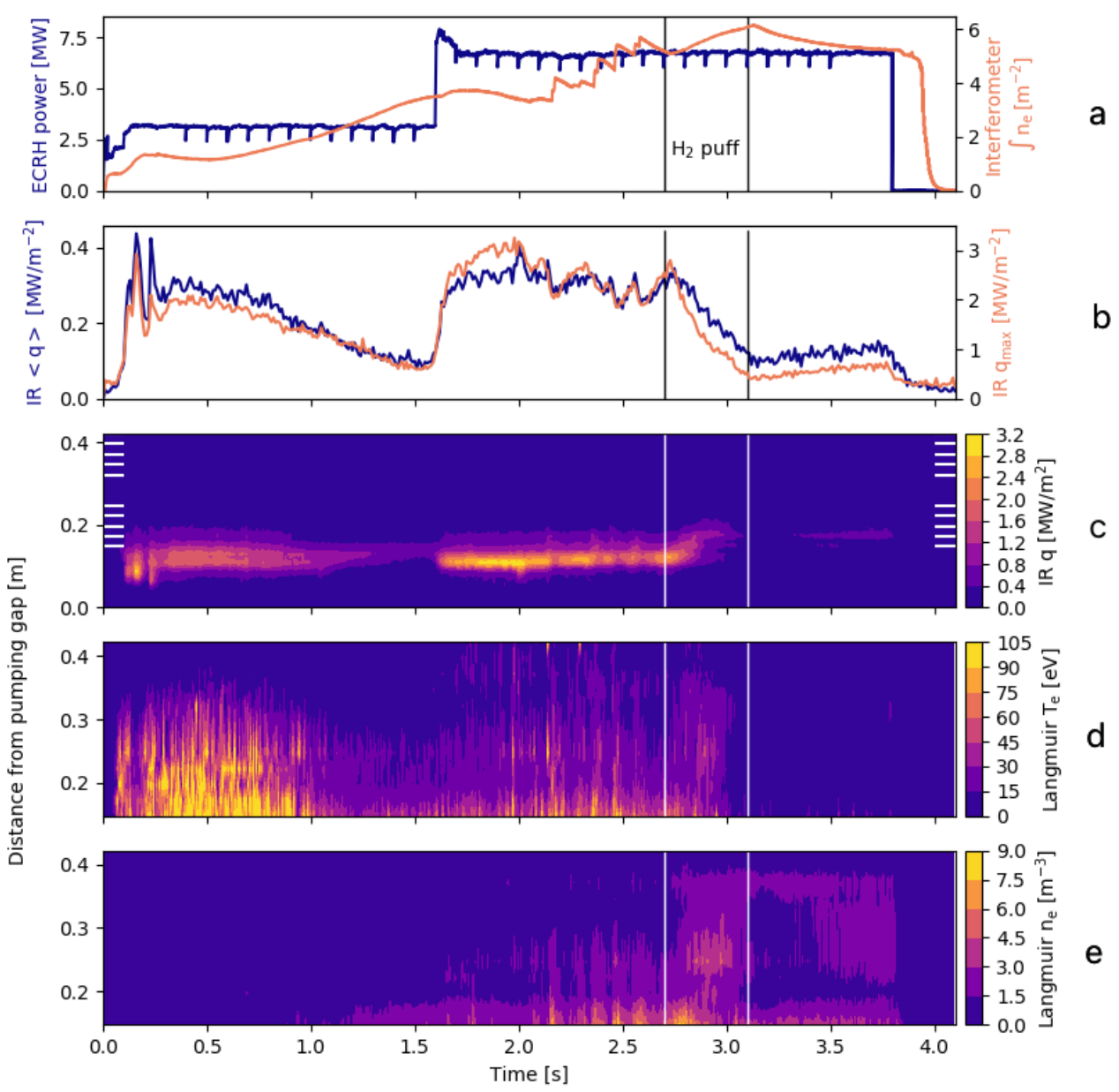

Fig. 5. The top trace (a) shows the ECRH heating power (deep purple) and the line-integrated plasma density in units of $10^{19} \mathrm{~m}^{-2}$ (orange). The next time traces (b) show the local peak heat flux (orange) and the average heat flux on the divertor targets (purple). Graph (c) shows the time evolution of the local heat flux across a divertor finger (finger 4 of the lower divertor in module 3). Graph (d) shows the electron temperature as determined by the array of Langmuir probes on the adjacent finger (their locations are indicated with white bars at the edges of graph (c)). Graph (e) shows the plasma density from the same Langmuir probes. The detachment starts at approximately $t=3.1 \mathrm{~s}$, and was triggered by the preceding divertor gas puff, which lasted from $t=2.7 \mathrm{~s}$ to $t=3.1 \mathrm{~s}$ ). Detachment was maintained stably until the programmed end of the discharge heating at $t=3.8 \mathrm{~s}$. As seen on graph (c) only one probe (no. 10) is at the strike line, and is only at the edge of it, at a distance from the pumping gap of $0.15 \mathrm{~m}$. Time traces of density and temperature derived from this probe are shown in fig. 6 (Shot 20171207.011)

For the shown discharge, a steady density rise leads to a heat flux reduction at 1.1-1.6 s, and the plasma may have detached soon thereafter, but the plasma reattaches after the power stepup at $\mathrm{t}=1.6 \mathrm{~s}$. The density starts rising again, due to pellet fueling, but the plasma remains attached, until the strong gas puff at $\mathrm{t}=2.7 \mathrm{~s}$ pushes the plasma into detachment, which lasts stably, without feedback control, until the plasma heating is terminated at $t=3.8 \mathrm{~s}$. The temperature measured by the Langmuir probes drops below $\sim 15 \mathrm{eV}$, whereas the density only temporarily rises during pellet injection and gas puff, but afterwards assumes the same level as before the pellet injection (see Fig. 5a). We note that the Langmuir probes are located in a different divertor unit and also in a different of the five edge island flux bundles than the one directly fuelled by the gas puff. 


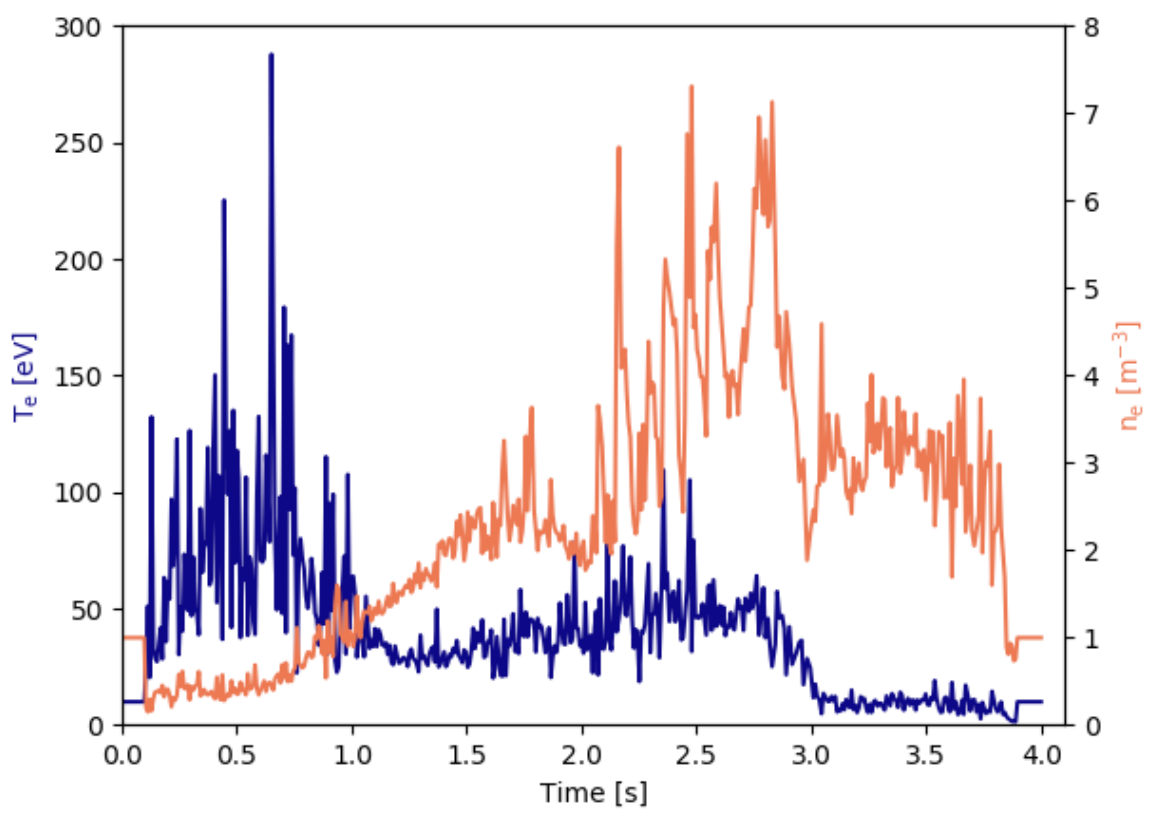

Fig. 6: Time traces of electron temperature $T_{e}$ (purple) and electron density $n_{e}$ (orange) calculated from the characteristics of probe no. 10, closest to the strike line for shot 20171207.011, also shown in Fig 5. Between $2.7 \mathrm{~s}$ and $3 \mathrm{~s}$, the electron temperature measured by this probe drops from $\sim 40 \mathrm{eV}$ to $\sim 10 \mathrm{eV}$, whereas the density returns to about the same level as before the start of pellet injection.

\subsection{Neutral gas pressure and exhaust}

Several pressure gauges of the ASDEX type have been installed in sub-divertor spaces and in midplane positions in order to characterize the exhaust capability and the neutral compression of the island divertor. Details can be found elsewhere[21].

Fig. 8 shows as an example the neutral pressure behavior in a discharge with pulsed divertor fueling (from $2-5 \mathrm{~s}$ ). The line-integrated plasma density is relatively low, $3 * 10^{19} \mathrm{~m}^{-2}$. The fueling pulses are clearly visible in the neutral pressure traces. The neutral pressure in the sub-divertor space varies between 3 and $5^{*} 10^{-5}$ mbar. The neutral compression, i.e. the ratio between sub-divertor and midplane pressure, varies between 6 and 8 , which is relatively small compared to the theoretical prediction of 180 [22]. However, those calculations were done for the case of a higher electron density. The observed subdivertor pressures and compression ratios indicate that for these plasmas the divertor does not act as the main sink for plasma particles: The subdivertor pressure is of order $5^{*} 10^{-5} \mathrm{mbar}$ (Fig. 7), and for this operation phase, the subdivertor volume was only pumped by turbopumps, giving an effective subdivertor pumping speed of $2.5^{*} 10^{4} 1 / \mathrm{s}$. This means that the particle removal rate was about $1.25 \mathrm{mbar} 1 / \mathrm{s}$ while the average external fueling rate lies at $6 \mathrm{mbar} 1 / \mathrm{s}$ which means $20 \%$ of the fueling rate was pumped by the turbopumps.

\subsection{Fueling and detachment}

A credible path to high performance in stellarators is to increase the plasma density. Often the achievable density, and the control of it, is limited by edge radiation instabilities that occur at low temperatures, usually referred to as MARFEs [23] and also seen in W7-X limiter operation [24]. Reducing the interaction between the edge plasma and edge neutrals often allows higher densities to be reached, and therefore localized and penetrating fueling methods 
such as pellets or supersonic gas injection with nozzles placed close to the last closed fluxsurface are advantageous. The pellet fueling system in W7-X for the operation phase OP1.2 was very successful in bringing up the density, but was limited to approximately 30 pellets per shot and was therefore unable to continuously fuel the discharges, which lasted up to tens of seconds already in OP1.2a and were extended up to $100 \mathrm{~s}$ in OP1.2b. The divertor gas fueling system was also operated with hydrogen towards the end of OP1.2a. It was able to increase density efficiently and trigger detachment, and also provide edge radiative cooling [25].

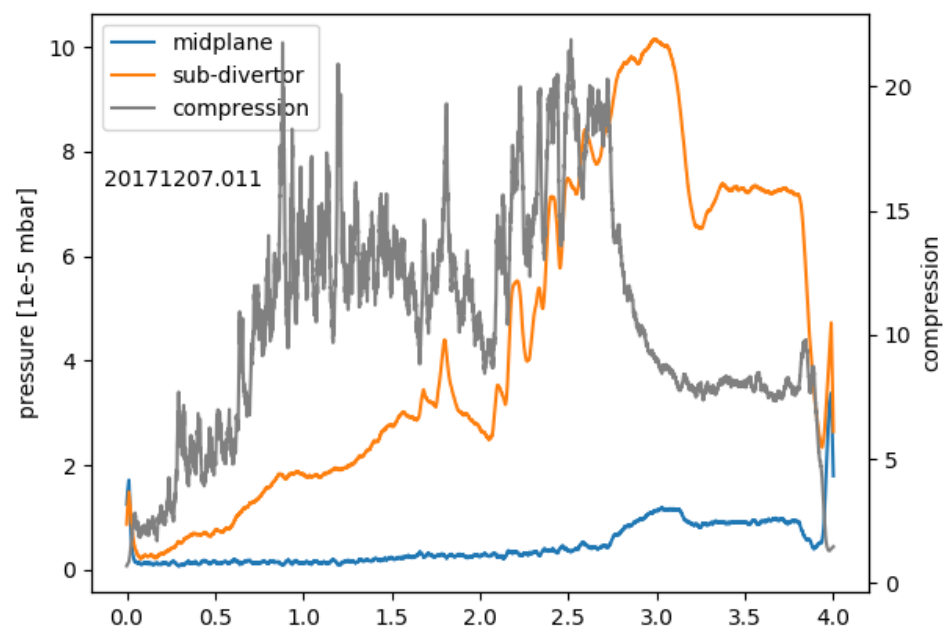

Figure 7. Neutral pressure traces of 20171207.011 and the neutral compression ratio for the same discharge shown in Figures 5 and 6 . The neutral compression ratio actually drops as the plasma enters detachment at $t=2.6 \mathrm{~s}$, to a level of about 7 or 8, from levels of 10-20 before detachment.

\section{PLASMA-WALL INTERACTIONS, WALL CONDITIONING AND IMPURITIES}

\subsection{Plasma-wall interactions and conditioning before boronization}

The plasma-facing components in OP1.2a and b were fine-grain graphite for all components with significant heat loads, including the divertor, the baffles, and the heat shields, and stainless steel panels for recessed areas. These components were in general not water-cooled but would warm up adiabatically during a pulse, and a general, slower increase of temperature during the run day was also observed, as one would expect. Before first operation in OP1.2a, a vacuum bakeout had been performed up to $150^{\circ} \mathrm{C}$ for more than one week, which eliminated most, but not all, of the water molecules trapped on the surfaces and in the bulk of the graphite. OP1.2a was performed without boronization, but extensive glowdischarge cleaning in hydrogen and helium was performed regularly between run days, and He discharges were used to help unload the walls from hydrogen during run days. While it took almost the entire OP1.1 campaign to get to reasonably low outgassing rates, the outgassing rate was below the best of OP1.1 after just two weeks of operation in OP1.2a. Nonetheless, absorption and release of hydrogen from the walls made it difficult to control the plasma density in hydrogen discharges, possibly also complicated by the fact that the main gas inlet valves are located several meters away from the plasma edge, recessed in long ports. Control of the density in helium discharges was unproblematic. 


\subsection{Effects of boronization on plasma performance}

In the OP1.2b phase, which started in July 2018, boronization was applied after the first few weeks of operation. This led to a strong reduction in oxygen outgassing. Fig. 8 (left) shows decreases in carbon and oxygen impurity concentrations of about one order of magnitude after the first boronization. The reduction of oxygen radiation in the plasma edge region is a direct result of the boronization, since boron chemically binds oxygen very effectively. The reduction of carbon is believed to be a consequence of the reduction of oxygen, which can act to strongly increase the chemical sputtering of carbon from the graphite PFCs [26].

The resulting order of magnitude reduction in edge impurity radiation allowed stable operation for a much larger range of hydrogen plasma densities - roughly a factor of three increase (Fig. 8 right, x-axis). As a result of the higher density, plasmas also exhibited increased confinement times (Fig. 8 right, y-axis), with the the data consistent with the ISS04 scaling predicting the confinement time to scale with $\mathrm{n}_{\mathrm{e}}{ }^{0.54}$. Thus, there is at this point no detectable direct improvement of the confinement time due to the cleaner plasmas, only the indirect one related to the larger density - also confirmed by the fact (partly visible in the figure) that plasmas at the same density and heating power had about the same confinement time before and after boronization. For a more detailed description of confinement time scalings, we refer to [27].
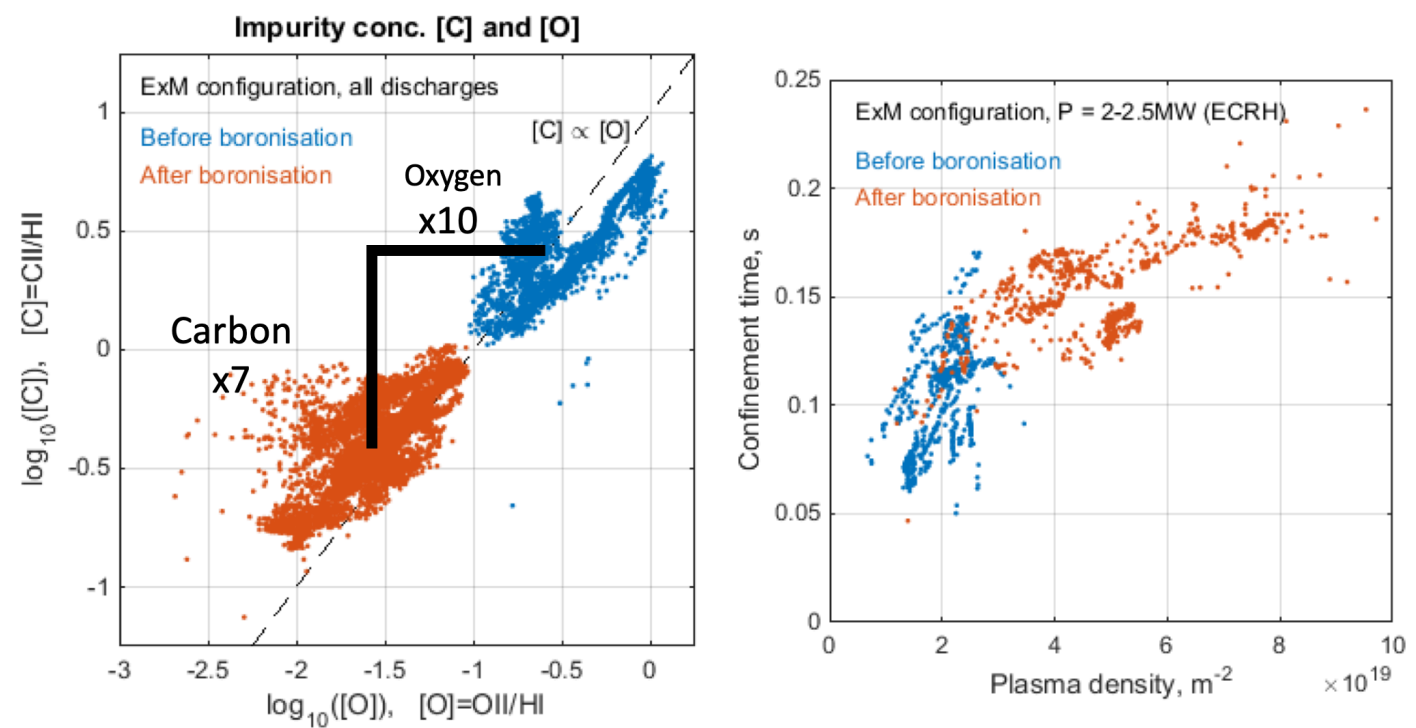

Figure 8. Left: The edge impurity concentrations were reduced by about a factor of 10 for oxygen and about a factor of 7 for carbon. Right: After boronization, stable plasma operation was extended to hydrogen densities about a factor of 3 larger than before boronization ( $x$-axis) and the confinement times also showed a modest increase, but no more than what was expected from the empirical scaling ISSO4.

\subsection{Spectroscopic observations of edge impurities before and after boronization}

Plasma and impurity lines were measured with visible divertor spectroscopy during OP1.2, mainly along lines of sight parallel and perpendicular to the divertor modules but also from the core plasma with lines of sight ending at the wall. Figure 9 shows an overview spectrum taken with an Echelle Esawin 3000 spectrometer during a hydrogen discharge, after boronization. In both cases, the core and divertor lines of sight, the Balmer lines are clearly visible. Among the impurity lines, carbon is the most intense, intrinsically being present in the edge plasma from the carbon target tiles. The C-III multiplet at $465 \mathrm{~nm}$ is particularly intense in both the divertor region and elsewhere in the plasma edge and is therefore also 
suitable for investigations with our ultra-high resolution spectrometer and Dopplercoherence-imaging diagnostics. Oxygen lines are present as well but much smaller.

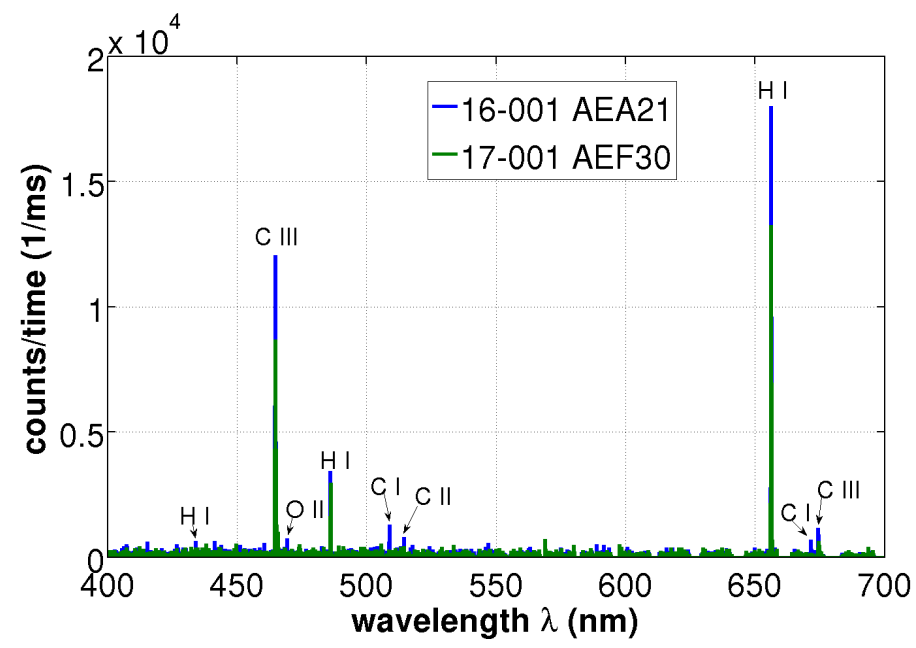

Figure 9: Visible spectra taken in W7-X at two different viewing ports: AEA21 with lines of sight viewing through the core plasma at the wall (20180905.16, blue line) and AEF30 with lines of sight viewing perpendicularly at the lower divertor module 3 (20180905.017, green line).

\subsection{Initial observations of a neutral-compressing divertor regime after boronization}

In addition to the already described reductions of edge radiation from oxygen and carbon, and the associated increased operation range for density in hydrogen plasmas, a new divertor detachment regime was also discovered after boronization. A detailed characterization and understanding of this regime is still evolving and will be published separately. The initial observations are presented here together with heuristic hypotheses about the differences between the two types of detachment, which can hopefully be confirmed after a careful analysis. The primary differences to the detachment described in Section 4 is that this new detachment has much higher divertor neutral compression (a factor of appr. 30, as opposed to a factor of appr. 7) and with that, a much higher divertor neutral pressure and exhaust rate (11.5-17 mbar 1/s, as opposed to $1.25 \mathrm{mbar} 1 / \mathrm{s})$, see Figure 10.
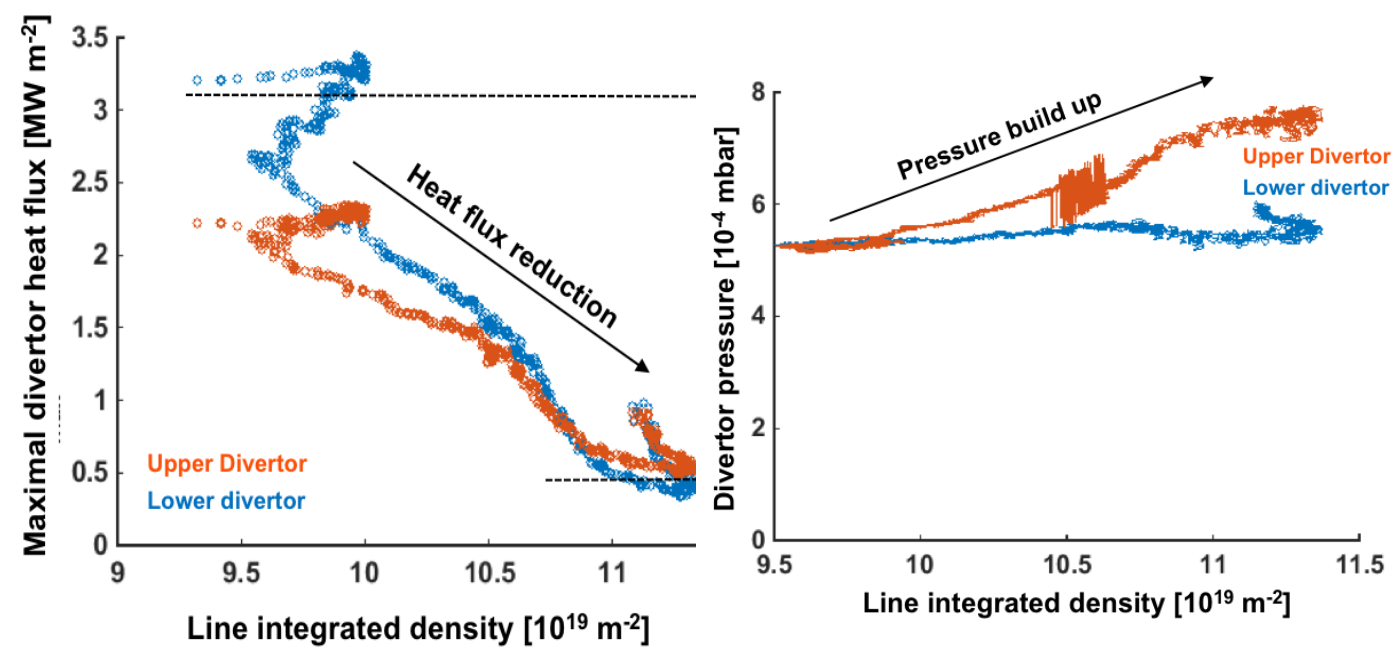
Figure 10: An example of detachment after boronization. Note that the x-axis is zoomed in to show the details of the detachment: The heat flux reduces by a factor of at least five for a $20 \%$ increase in line-integrated plasma density (left). The subdivertor neutral pressure shows a more or less proportional increase, but importantly, is an order of magnitude higher than for the detachment experiments before boronization (Section 4).

\subsection{Heuristic explanation for the differences in the neutral compression of detached discharges before and after boronization}
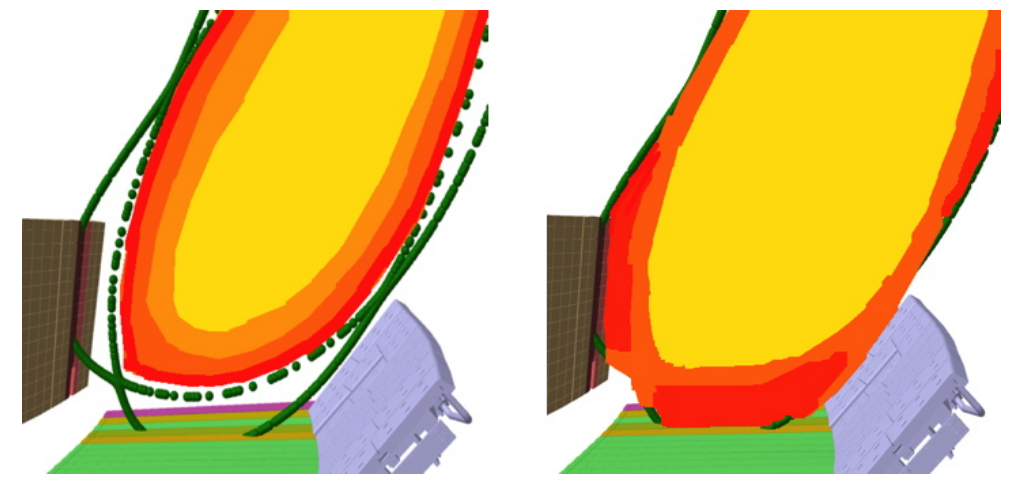

Figure 11: We illustrate here our hypothesis for the large difference in divertor neutral compression between the preboronization detachment (left) and post-boronization detachment (right). At higher heating power and reduced $C$ - and $O$ radiation, the plasma more fully "plugs" the divertor and allows a better neutral compression. Also, the neutral source (presumably due to charge exchange) would be distributed rather evenly on the magnetic surface before boronization, whereas after, the neutral source would be more concentrated in the divertor region itself, and could include three-body recombination in this case. The sketched colors are meant to illustrate hot core plasma (yellow), cooler edge plasma (orange) and rather cold outer edge plasma (red).

We present here potential explanations for the large difference between the neutral compression ratios of the two kinds of detachment observed, illustrated in Figure 11. First, we note that the low-power, low-neutral-compression detachment was observed much more readily before boronization than after, and that the higher-power, high-neutral-compression detachment was only observed after boronisation. This suggests that the low-power detachment is directly related to oxygen and carbon radiation. At lower heating powers before boronization, a distributed edge radiating mantle of hydrogen, carbon and oxygen could dissipate the particle energy. The edge island structure would not be sufficiently filled with plasma to re-ionize neutrals that move along the divertor baffle structures. That is, the plasma would not fill and plug the divertor fully. Another reason why the neutral compression is low could be that the source of neutrals is due to neutralization in the whole outer mantle, overall in the device, ie. not as concentrated in the divertor region as expected. After boronization, at higher hydrogen densities and heating powers, and with the strongly reduced carbon and oxygen content, the outflowing core plasma fills the island structure and "plugs" the divertor better, preventing neutrals from leaving the divertor region by reionizing them and dragging them back into the divertor region. A recombination zone can in this case develop in the divertor region, presumably close to the divertor plates.

At this point, there is only preliminary evidence for this physical picture, but also no evidence clearly against it. We show in Figure 12 an example of preliminary evidence - visible light images for the two different types of detachment taken with one of the EDICAM cameras of the video surveillance system [28]. Figure 13a and b show the pre-boronization and postboronization pictures respectively. 


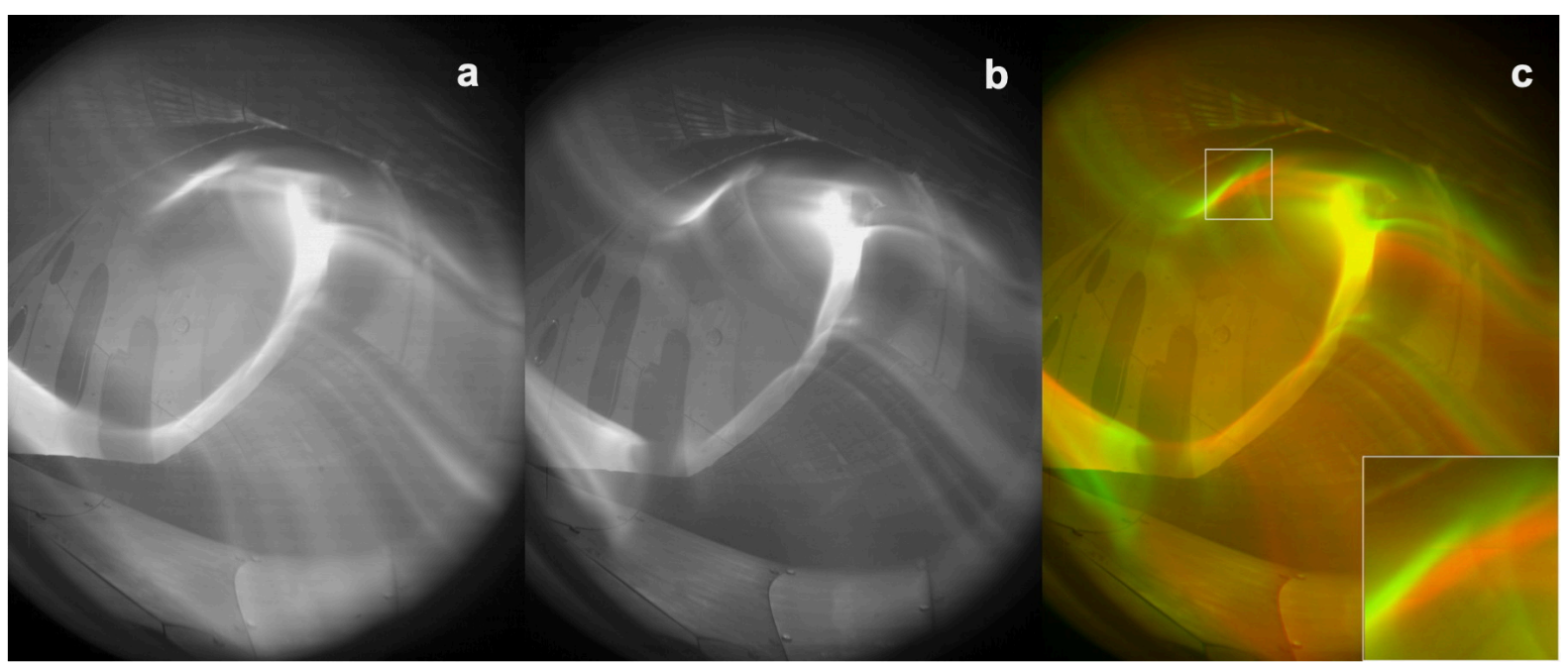

Figure 12. Visible radiation from the plasma edge from the EDICAM video diagnostic system on W7-X. Left (a): Shot 20171109045 at $t=2.50 \mathrm{~s}$ (pre-boronization detachment). Middle (b): Shot $20180814024 t=7.62 \mathrm{~s}$ (post-boronization detachment). Right (c): The two photos added in separate colour channels (red: pre-boronization, green: post-boronization). The pre-boronization detachment shows a more poloidally distributed radiation, and also radiation slightly deeper inside. One particularly clear example of the latter is shown as an insert magnified 3 times at the bottom right of the figure.

The more poloidally distributed radiation for pre-boronization is visible by comparison between Fig 12a and 12b, consistent with the radiating mantle hypothesis. The minor-radial location of the radiation zone, and the difference in divertor plugging, which is what is illustrated in the cartoons in Fig 11, is not obvious. It can only be seen in Figure 12c, which combines the two images a and $b$, by assigning separate colour channels to the two images, red and green: Red indicates where the radiation is stronger in the pre-boronization picture, and green where the radiation is stronger in the post-boronization picture, and yellow indicates where both radiate with about the same intensity. A tendency for a red colour deeper inside, towards the core plasma region, and the green colour further outside can be seen, in particular at the tangential view areas, where the line-integrating nature of the measurement combined with the three-dimensional geometry makes this comparison less difficult. One such area is zoomed in for clarity, at the bottom right part of the figure. This lends some credibility to the divertor-plugging hypothesis. The colour picture also visualizes other differences, to be analysed in future work. Future work will also aim to clarify the validity of these two proposed hypotheses (both of which could be at play simultaneously), as well as the origins of the recycling neutrals in the main chamber.

\subsection{Summary}

First operation with an island divertor in W7-X has brought many encouraging results. The heat loads generally appeared as expected, and for high-density hydrogen plasmas, stable heat-flux detachment was achieved under several conditions. The heat flux reduction was at least a factor of 10, and was characterized by a significant drop in target electron temperature and no large increases in the target densities, unlike typical tokamak-detachment cases. Likely related to this lack of density increase, the detachment observed in OP1.2a was characterized by only a modest neutral compression ratio of about 7 .

High-density operation was challenging in hydrogen plasmas throughout OP1.2a but was achieved successfully after the first boronization in OP1.2b. This also led to detachment with a much higher neutral compression, about 30 . Two potential explanations for the differences 
in the neutral compression ratio for these two detachment regimes were proposed, one related to plugging efficiency, the other that the pre-boronization detachment is more akin to a radiating mantle than a classical tokamak divertor detachment.

\section{ACKNOWLEDGEMENTS}

This work has been carried out within the framework of the EUROfusion Consortium and has received funding from the Euratom research and training programme 2014-2018 and 2019-2020 under grant agreement No 633053. The views and opinions expressed herein do not necessarily reflect those of the European Commission. This work was supported in part by the U.S. Department of Energy (DoE) under grant DE-SC0014210 and by discretional funding of the Department of Engineering Physics and the College of Nuclear Engineering at the University of Wisconsin - Madison, USA.

\section{REFERENCES}

[1] T. Klinger et al. Plasma Phys. Controlled Fusion 59(1) 014018 (2017)

[2] H.-S. Bosch et al., Nuclear Fusion 57, 116015 (2017)

[3] R. C. Wolf et al., Nuclear Fusion 57102020 (2017)

[4] T. Sunn Pedersen et al., Physics of Plasmas 24055503 (2017)

[5] T. Klinger et al., IAEA conference 2018, submitted to Nuclear Fusion (2019)

[6] M. Hirsch et al., Plasma Phys. Controlled Fusion (2008)

[7] S. Lazerson, et al.. Plasma Phys. Controlled Fusion, 60 (12), 124002-12. http://doi.org/10.1088/1361-6587/aae96b (2018)

[8] S. A. Bozhenkov et al., Measurements and correction of the $1 / 1$ error field in Wendelstein 7-X. Nuclear Fusion, 59(2), 026004-12. http://doi.org/10.1088/1741-4326/aaf20c (2018)

[9] B. Sieglin, et al., Review of Scientific Instruments 86, 113502 (2015)

[10] C. Killer et al., IAEA conference 2018, submitted to Nuclear Fusion (2019)

[11] T. Eich et al., Nucl. Fusion 53093031 (2013)

[12] R. J. Goldston, Nucl. Fusion 52013009 (2012)

[13] P. Sinha et al., Nucl. Fusion 57016027 (2017)

[14] H. Niemann et al., PRL, in preparation

[15] J.D. Lore, et al., IEEE TPS 42, 539 (2014).

[16] J. Geiger, et al., Contrib. Plasma Phys., 50 (2010) 770

[17] H. Hölbe, et al., Nucl. Fusion 56, 026015, 2016.

[18] M. Jakubowski, et al., Rev. Sci. Inst. 89 10E116 (2018)

[19] J.D. Lore, et al., IEEE TPS 46 (2018) 1387.

[20] T. Sunn Pedersen, et al., "Confirmation of the topology of the Wendelstein 7-X magnetic field to better than 1:100,000”, Nature Communications 713493 (2016), https://www.nature.com/articles/ncomms13493

[21] U. Wenzel et al 2017 JINST 12 C09008

[22] D. Sharma et al, Optimization of the W7-X pumping system, Proceedings of the EPS conference 2003, P-1.14

[23] U. Samm et al., J. Nucl. Mater. P. 266666 (1999)

[24] U. Wenzel et al., Nucl. Fusion 58096025 (2018)

[25] F. Effenberg et al., IAEA conference 2018, submitted to Nuclear Fusion (2019)

[26] R. Brakel et al., IAEA conference 2018, submitted to Nuclear Fusion (2019)

[27] G. Fuchert et al, IAEA conference 2018, submitted to Nuclear Fusion (2019)

[28] S. Zoletnik, T. Szabolics, G. Kocsis, T.Szepesi, D.Dunai, Fusion Engineering and Design 88 p. 1405 (2013) 